\title{
Plug-and-play distributed state estimation for linear systems*
}

\author{
Stefano Riverso†, Marcello Farina $\ddagger$ Riccardo Scattolini \\ Ferrari-Trecate \\ Dipartimento di Ingegneria Industriale e dell'Informazione \\ Università degli Studi di Pavia
}

Technical Report

September, 2013

\begin{abstract}
This paper proposes a state estimator for large-scale linear systems described by the interaction of state-coupled subsystems affected by bounded disturbances. We equip each subsystem with a Local State Estimator (LSE) for the reconstruction of the subsystem states using pieces of information from parent subsystems only. Moreover we provide conditions guaranteeing that the estimation errors are confined into prescribed polyhedral sets and converge to zero in absence of disturbances. Quite remarkably, the design of an LSE is recast into an optimization problem that requires data from the corresponding subsystem and its parents only. This allows one to synthesize LSEs in a Plug-and-Play (PnP) fashion, i.e. when a subsystem gets added, the update of the whole estimator requires at most the design of an LSE for the subsystem and its parents. Theoretical results are backed up by numerical experiments on a mechanical system.
\end{abstract}

\footnotetext{
*The research leading to these results has received funding from the European Union Seventh Framework Programme [FP7/2007-2013] under grant agreement $n^{\circ} 257462$ HYCON2 Network of excellence.

${ }^{\dagger}$ Electronic address: stefano.riverso@unipv.it; Corresponding author

‡Electronic address: farina@elet.polimi.it

§Electronic address: riccardo.scattolini@elet.polimi.it

"Electronic address: giancarlo.ferrari@unipv.it

S. Riverso and G. Ferrari-Trecate are with Dipartimento di Ingegneria Industriale e dell'Informazione, Università degli Studi di Pavia, via Ferrata 1, 27100 Pavia, Italy

M. Farina and R. Scattolini are with Dipartimento di Elettronica e Informazione, Politecnico di Milano, via Ponzio 34/5, 20133 Milan, Italy
} 


\section{Introduction}

In several applications, the use of centralized state estimators is hampered by the complexity of the underlying systems. As an example, when plants are composed by several subsystems arranged in a parent-child coupling relation, online operations, such as the transmission of output samples to a central processing unit or the simultaneous estimation of all states, can be prohibitive. This has motivated a large body of research on Distributed State Estimators (DSEs) where subsystems are equipped with LSEs connected through a communication network and dedicated to the reconstruction of local states only [1, 2, 3, 4, 5, 6, 7, 8, Concerning the required communication links, some methods are more parsimonious as they do not need information to be exchanged between all LSEs, but only along the edges of a directed network with the parent-child topology induced by subsystems coupling [3, 4, 5, 6, 7, 8, Furthermore, there are methods that also guarantee the fulfillment of constraints on local states [6] or estimation errors [7, 8].

As in [7] and [8], in this paper we consider discrete-time linear time-invariant subsystems affected by bounded disturbances and propose a DSE composed by LSEs with a Luenberger-like structure and connected through a network with parent-child topology. We provide conditions for guaranteeing estimation errors fulfill prescribed polyhedral constraints at all times and converge to zero when there are no disturbances. A key feature of our approach is that, differently from [7] and [8], checking these conditions amounts to numerical tests that are associated with individual LSEs and that can be conducted in parallel using hardware collocated with subsystems. Furthermore, each test requires data from parent subsystems only. These properties enable PnP design of LSEs, meaning that (i) when a subsystem is added to a plant, the corresponding LSE can be designed using pieces of information from parent subsystems only; (ii) in order to preserve the key properties of the whole DSE, the plugging in and out of a subsystem triggers at most the update of LSEs associated to child subsystems and (iii) the design/update of an LSE is automatized, e.g. it is recast into an optimization problem that can be solved using local hardware. We highlight that addition and removal of subsystems, as well as synthesis of LSEs, are here considered as offline operations and therefore no hybrid dynamics is generated. Our method, that parallels the PnP procedure for the design of decentralized model predictive controllers proposed in [9] and [10], can be useful in the context of systems of systems [11 and cyber-physical systems [12 where, typically, the number of subsystems changes over time.

The paper is structured as follows. The DSE is introduced in Section 2, In Section 3 the main results allowing design decentralization are presented together with the optimization-based synthesis of LSEs. PnP operations are discussed in 4 In Section 5 we illustrate the use of the DSE for reconstructing the states of a 2D array of masses connected by springs and dampers. Finally, Section 6 is devoted to conclusions.

Notation. We use $a: b$ for the set of integers $\{a, a+1, \ldots, b\}$. The symbol $\mathbb{R}_{+}^{n}$ stands for the vectors in $\mathbb{R}^{n}$ with nonnegative elements. The column vector with $s$ components $v_{1}, \ldots, v_{s}$ is $\mathbf{v}=\left(v_{1}, \ldots, v_{s}\right)$. The symbol $\oplus$ denotes the Minkowski sum, i.e. $A=B \oplus C$ if and only if $A=\{a: a=b+c, b \in B, c \in C\}$. Moreover, $\bigoplus_{i=1}^{s} G_{i}=G_{1} \oplus \ldots \oplus G_{s}$. The symbol $\mathbf{1}_{\alpha}$ (resp. $\mathbf{0}_{\alpha}$ ) denotes a column vector with $\alpha \in \mathbb{N}$ elements all equal to 1 (resp. 0 ). Given a matrix $A \in \mathbb{R}^{n \times n}$, with entries $a_{i j}$ its entry-wise 1 -norm is $\|A\|_{1}=\sum_{i=1}^{n} \sum_{j=1}^{n}\left|a_{i j}\right|$ and its Frobenius norm is $\|A\|_{F}=\sqrt{\sum_{i=1}^{n} \sum_{j=1}^{n} a_{i j}^{2}}$. The standard Euclidean norm is denoted with $\|\cdot\|$. The pseudo-inverse of a matrix $A \in \mathbb{R}^{m \times n}$ is denoted with $A^{b}$.

The set $\mathbb{X} \subseteq \mathbb{R}^{n}$ is positively invariant [13] for $x(t+1)=f(x(t))$, if $x(t) \in \mathbb{X} \Rightarrow f(x(t)) \in \mathbb{X}$. 
The set $\mathbb{X} \subseteq \mathbb{R}^{n}$ is Robust Positively Invariant (RPI) [13] for $x(t+1)=f(x(t), w(t)), w(t) \in \mathbb{W} \subseteq$ $\mathbb{R}^{m}$ if $x(t) \in \mathbb{X} \Rightarrow f(x(t), w(t)) \in \mathbb{X}, \forall w(t) \in \mathbb{W}$. The RPI set $\overline{\mathbb{X}}$ is maximal (MRPI) if every other RPI $\mathbb{X}$ verifies $\overline{\mathbb{X}} \supseteq \mathbb{X}$. The RPI set $\underline{\mathbb{X}}$ is minimal (mRPI) if every other RPI $\mathbb{X}$ verifies $\underline{\mathbb{X}} \subseteq \mathbb{X}$. The RPI set $\mathbb{X}(\epsilon)$ is a $\epsilon$-outer approximation of the mRPI $\mathbb{X}$ if

$$
x \in \mathbb{X}(\epsilon) \Rightarrow \exists \underline{x} \in \underline{\mathbb{X}} \text { and } \tilde{x} \in B_{\epsilon}(0): x=\underline{x}+\tilde{x}
$$

where, for $\epsilon>0, B_{\epsilon}(v)=\left\{x \in \mathbb{R}^{n} \mid\|x-v\|<\epsilon\right\}$.

\section{Distributed state estimator}

We consider a discrete-time Linear Time Invariant (LTI) system

$$
\begin{aligned}
\mathbf{x}^{+} & =\mathbf{A x}+\mathbf{B u}+\mathbf{D w} \\
\mathbf{y} & =\mathbf{C x}
\end{aligned}
$$

where $\mathbf{x} \in \mathbb{R}^{n}, \mathbf{u} \in \mathbb{R}^{m}, \mathbf{y} \in \mathbb{R}^{p}$ and $\mathbf{w} \in \mathbb{R}^{r}$ are the state, the input, the output and the disturbance, respectively, at time $t$ and $\mathbf{x}^{+}$stands for $\mathbf{x}$ at time $t+1$. The state is composed by $M$ state vectors $x_{[i]} \in \mathbb{R}^{n_{i}}, i \in \mathcal{M}=1: M$ such that $\mathbf{x}=\left(x_{[1]}, \ldots, x_{[M]}\right)$, and $n=\sum_{i \in \mathcal{M}} n_{i}$. Similarly, the input, the output and the disturbance are composed by $M$ vectors $u_{[i]} \in \mathbb{R}^{m_{i}}$, $y_{[i]} \in \mathbb{R}^{p_{i}}, w_{[i]} \in \mathbb{R}^{r_{i}}, i \in \mathcal{M}$ such that $\mathbf{u}=\left(u_{[1]}, \ldots, u_{[M]}\right), m=\sum_{i \in \mathcal{M}} m_{i}, \mathbf{y}=\left(y_{[1]}, \ldots, y_{[M]}\right)$, $p=\sum_{i \in \mathcal{M}} p_{i}, \mathbf{w}=\left(w_{[1]}, \ldots, w_{[M]}\right)$ and $r=\sum_{i \in \mathcal{M}} r_{i}$.

We assume (11) can be equivalently described by subsystems $\Sigma_{[i]}, i \in \mathcal{M}$, given by

$$
\begin{aligned}
\Sigma_{[i]}: \quad x^{+}{ }_{[i]} & =A_{i i} x_{[i]}+B_{i} u_{[i]}+\sum_{j \in \mathcal{N}_{i}} A_{i j} x_{[j]}+D_{i} w_{[i]} \\
y_{[i]} & =C_{i} x_{[i]}
\end{aligned}
$$

where $A_{i j} \in \mathbb{R}^{n_{i} \times n_{j}}, i, j \in \mathcal{M}, B_{i} \in \mathbb{R}^{n_{i} \times m_{i}}, D_{i} \in \mathbb{R}^{n_{i} \times r_{i}}, C_{i} \in \mathbb{R}^{p_{i} \times n_{i}}$ and $\mathcal{N}_{i}$ is the set of parents of subsystem $i$ defined as $\mathcal{N}_{i}=\left\{j \in \mathcal{M}: A_{i j} \neq 0, i \neq j\right\}$. Moreover, since $y_{[i]}$ depends on the local state $x_{[i]}$ only, subsystems $\Sigma_{[i]}$ are output-decoupled and then $\mathbf{C}=\operatorname{diag}\left(C_{1}, \ldots, C_{M}\right)$. Similarly, subsystems $\Sigma_{[i]}$ are input- and disturbance-decoupled, i.e. $\mathbf{B}=\operatorname{diag}\left(B_{1}, \ldots, B_{M}\right)$ and $\mathbf{D}=\operatorname{diag}\left(D_{1}, \ldots, D_{M}\right)$. We also assume

$$
w_{[i]} \in \mathbb{W}_{i}
$$

where the set $\mathbb{W}_{i} \subset \mathbb{R}^{r_{i}}$ is a zonotope centered at the origin, i.e. a polytope that is centrally symmetric about the origin. Without loss of generality, $\mathbb{W}_{i}$ can be written as

$$
\begin{aligned}
\mathbb{W}_{i} & =\left\{w_{[i]} \in \mathbb{R}^{r_{i}} \mid \mathcal{F}_{i} w_{[i]} \leq \mathbf{1}_{\bar{v}_{i}}\right\} \\
& =\left\{w_{[i]} \in \mathbb{R}^{r_{i}} \mid w_{[i]}=\Delta_{i} l_{i},\left\|l_{i}\right\|_{\infty} \leq 1\right\}
\end{aligned}
$$

where $\mathcal{F}_{i}=\left(f_{i, 1}^{T}, \ldots, f_{i, \bar{v}_{i}}^{T}\right) \in \mathbb{R}^{\bar{v}_{i} \times r_{i}}, \operatorname{rank}\left(\mathcal{F}_{i}\right)=r_{i}, \Delta_{i} \in \mathbb{R}^{r_{i} \times \bar{r}_{i}}$ and $l_{i} \in \mathbb{R}^{\bar{r}_{i}}$.

In this section we propose a Distributed State Estimator (DSE) for (11). As in [7] and [8], we define for $i \in \mathcal{M}$ the Local State Estimator (LSE)

$$
\begin{array}{r}
\tilde{\Sigma}_{[i]}: \quad \tilde{x}_{[i]}^{+}=A_{i i} \tilde{x}_{[i]}+B_{i} u_{[i]}-L_{i i}\left(y_{[i]}-C_{i} \tilde{x}_{[i]}\right)+ \\
\sum_{j \in \mathcal{N}_{i}} A_{i j} \tilde{x}_{[j]}-\sum_{j \in \mathcal{N}_{i}} \delta_{i j} L_{i j}\left(y_{[j]}-C_{j} \tilde{x}_{[j]}\right)
\end{array}
$$


where $\tilde{x}_{[i]} \in \mathbb{R}^{n_{i}}$ is the state estimate, $L_{i j} \in \mathbb{R}^{n_{i} \times p_{j}}$ are gain matrices and $\delta_{i j} \in\{0,1\}$. This implies that $\tilde{\Sigma}_{[i]}$ depends only on local variables $\left(\tilde{x}_{[i]}, u_{[i]}\right.$ and $\left.y_{[i]}\right)$ and parents' variables $\left(\tilde{x}_{[j]}\right.$ and $\left.y_{[j]}, j \in \mathcal{N}_{i}\right)$. Binary parameters $\delta_{i j}, j \in \mathcal{N}_{i}$ can be chosen equal to one for exploiting the knowledge of parents' outputs, or equal to zero for reducing the number of transmitted output samples.

Defining the state estimation error as

$$
e_{[i]}=x_{[i]}-\tilde{x}_{[i]},
$$

from (2), (5) and (6), we obtain the local error dynamics

$$
\Theta_{[i]}: \quad e_{[i]}^{+}=\bar{A}_{i i} e_{[i]}+\sum_{j \in \mathcal{N}_{i}} \bar{A}_{i j} e_{[j]}+D_{i} w_{[i]}
$$

where $\bar{A}_{i i}=A_{i i}+L_{i i} C_{i}$ and $\bar{A}_{i j}=A_{i j}+\delta_{i j} L_{i j} C_{j}, i \neq j$. Our main goal is to solve the following problem.

Problem 1. Design in a decentralized fashion LSEs $\tilde{\Sigma}_{[i]}, i \in \mathcal{M}$ that

(a) are nominally convergent, i.e. when $\mathbb{W}_{i}=\{0\}$ it holds

$$
\left\|e_{[i]}(t)\right\| \rightarrow 0 \text { as } t \rightarrow \infty
$$

(b) guarantee, for suitable initial conditions

$$
e_{[i]}(t) \in \mathbb{E}_{i}, \forall t \geq 0
$$

where $\mathbb{E}_{i} \subseteq \mathbb{R}^{n_{i}}$ are zonotopes centered at the origin given by

$$
\begin{aligned}
\mathbb{E}_{i} & =\left\{e_{[i]} \in \mathbb{R}^{n_{i}} \mid \mathcal{H}_{i} e_{[i]} \leq 1_{\bar{\tau}_{i}}\right\} \\
& =\left\{e_{[i]} \in \mathbb{R}^{n_{i}} \mid e_{[i]}=\Xi_{i} d_{i},\left\|d_{i}\right\|_{\infty} \leq 1\right\}
\end{aligned}
$$

In (10), $\mathcal{H}_{i}=\left(h_{i, 1}^{T}, \ldots, h_{i, \bar{\tau}_{i}}^{T}\right) \in \mathbb{R}^{\bar{\tau}_{i} \times n_{i}}, \operatorname{rank}\left(\mathcal{H}_{i}\right)=n_{i}, \Xi_{i} \in \mathbb{R}^{n_{i} \times \bar{n}_{i}}$ and $d_{i} \in \mathbb{R}^{\bar{n}_{i}}$.

Defining the variable $\mathbf{e}=\left(e_{[1]}, \ldots, e_{[M]}\right) \in \mathbb{R}^{n}$, from (7) one obtains the collective dynamics of the estimation error

$$
\mathbf{e}^{+}=\overline{\mathbf{A}} \mathbf{e}+\mathbf{D w}
$$

where the matrix $\overline{\mathbf{A}}$ is composed by blocks $\bar{A}_{i j}, i, j \in \mathcal{M}$.

We equip system (11) with constraints $\mathbf{e} \in \mathbb{E}=\prod_{i \in \mathcal{M}} \mathbb{E}_{i}$ and $\mathbf{w} \in \mathbb{W}=\prod_{i \in \mathcal{M}} \mathbb{W}_{i}$.

Let $\mathbf{L}$ be the matrix composed by blocks $L_{i j}, i, j \in \mathcal{M}$. From (11), if $\mathbf{L}$ is such that $\overline{\mathbf{A}}$ is Schur, then property (8) holds. Moreover, if there exists an RPI set $\mathbb{S} \subseteq \mathbb{E}$ for the constrained system (11), then $\mathbf{e}(0) \in \mathbb{S}$ guarantees property (91). We highlight that methods based on Linear Programming (LP) for computing $\mathbb{S}$ exist $[14,15$. However the resulting LP problems require the knowledge of the collective model (10) and therefore they become prohibitive for large-scale systems.

In absence of coupling between subsystems (i.e. $A_{i j}=0, i \neq j$ ) the error dynamics (7) are decoupled as well. Therefore, from (11), if $L_{i i}$ are such that matrices $\bar{A}_{i i}$ are Schur, then (8) holds. Furthermore, if there is an RPI set $\mathbb{S}_{i} \subseteq \mathbb{E}_{i}$ for each local error dynamics, property (9) can be guaranteed by requiring $e_{[i]}(0) \in \mathbb{S}_{i}$. Since $\mathbb{E}_{i}$ and $\mathbb{W}_{i}$ are polytopes, using the algorithms in 
[14, 15] the computation of sets $\mathbb{S}_{i}, i \in \mathcal{M}$ requires the solution of $M$ LP problems that can be solved in parallel using computational resources collocated with subsystems.

In the next section we propose a method for bridging the gap between the two extreme cases described above, i.e. for designing LSEs in a decentralized fashion even in presence of coupling between subsystems.

\section{Decentralization of LSE design}

In the following, we first solve Problem $\mathbb{1}$ in the case of $\mathbb{W}=\{0\}$ i.e. no disturbances act on subsystems (10), and then show how to take disturbances into account.

When $\mathbb{W}=\{0\}$, we need to find matrices $L_{i j} i, j \in \mathcal{M}$ such that system (11) is asymptotically stable. To achieve this aim in a decentralized fashion, we treat the coupling term $v_{[i]}=\sum_{j \in \mathcal{N}_{i}} \bar{A}_{i j} e_{[j]}$ as a disturbance for the error dynamics

$$
e_{[i]}^{+}=\bar{A}_{i i} e_{[i]}+v_{[i]}
$$

and then confine the error into an RPI set $\mathbb{S}_{i} \subseteq \mathbb{E}_{i}$ for (12) and $v_{[i]} \in \mathbb{V}_{i}=\bigoplus_{j \in \mathcal{N}_{i}} \bar{A}_{i j} \mathbb{E}_{j}$. The main result, that will also enable PnP design of LSEs, is given in the next proposition.

Proposition 1. Let $\mathbb{W}=\{0\}$. If, for given matrices $L_{i j}$ and parameters $\delta_{i j}, i, j \in \mathcal{M}$, the following conditions are fulfilled

$$
\begin{aligned}
& \bar{A}_{i i} \text { is Schur, } \forall i \in \mathcal{M} \\
& \beta_{i}=\sum_{j \in \mathcal{N}_{i}} \sum_{k=0}^{\infty}\left\|\mathcal{H}_{i} \bar{A}_{i i}^{k} \bar{A}_{i j} \mathcal{H}_{j}^{b}\right\|_{\infty}<1, \forall i \in \mathcal{M}
\end{aligned}
$$

then

(I) $\overline{\mathbf{A}}$ is Schur;

(II) $\forall i \in \mathcal{M}$ there exists an RPI $\mathbb{S}_{i} \subseteq \mathbb{E}_{i}$ for dynamics (12), such that $\mathbb{S}=\prod_{i \in \mathcal{M}} \mathbb{S}_{i}$ is a positively invariant set for system (11).

Proof. The proof is given in the Appendix 7.1

Some comments are in order. The conditions in Proposition—guarantee that if $e_{[i]}(0) \in \mathbb{S}_{i}, \forall i \in$ $\mathcal{M}$, then (8) and (9) hold. Condition (13b), that stems from the small gain theorem for networks [16], implies that the coupling between subsystems must be sufficiently small. In particular, if subsystems are decoupled, (13b) is always fulfilled and nominal convergence of the state estimator is guaranteed by condition (13a) only.

Remark 1. We highlight that, for a given $i \in \mathcal{M}$, the quantity $\beta_{i}$ in (13) depends only upon local fixed parameters $\left\{A_{i i}, C_{i}, \mathcal{H}_{i}\right\}$, neighbors' fixed parameters $\left\{A_{i j}, C_{j}, \mathcal{H}_{j}\right\}_{j \in \mathcal{N}_{i}}$ and local tunable parameters $\left\{L_{i i},\left\{L_{i j}, \delta_{i j}\right\}_{j \in \mathcal{N}_{i}}\right\}$ but not on neighbors' tunable parameters. This implies that the choice of $\left\{L_{i i},\left\{L_{i j}, \delta_{i j}\right\}_{j \in \mathcal{N}_{i}}\right\}$ does not influence the choice of $\left\{L_{j j},\left\{L_{j k}, \delta_{j k}\right\}_{k \in \mathcal{N}_{j}}\right\}$, for $i \neq j$.

When system (11) is affected by disturbances, i.e. $\mathbb{W} \neq\{0\}$, we can still use (13) for guaranteeing the stability of matrix $\overline{\mathbf{A}}$, but we need an additional condition in order to guarantee the existence of an RPI set $\mathbb{S}_{i} \subseteq \mathbb{E}_{i}$ for the error dynamics

$$
e_{[i]}^{+}=\bar{A}_{i i} e_{[i]}+\tilde{v}_{[i]}
$$


where the disturbance $\tilde{v}_{[i]}$ verifies

$$
\tilde{v}_{[i]}=v_{[i]}+D_{i} w_{[i]} \in \tilde{\mathbb{V}}_{i}=\left(\bigoplus_{j \in \mathcal{N}_{i}} \bar{A}_{i j} \mathbb{E}_{j} \oplus D_{i} \mathbb{W}_{i}\right)
$$

Since $\tilde{\mathbb{V}}_{i}$ is a zonotope, it can be written as $\tilde{\mathbb{V}}_{i}=\left\{\tilde{v}_{[i]} \in \mathbb{R}^{\tilde{n}_{i}} \mid \tilde{v}_{[i]}=\Psi_{i} \tilde{d}_{i},\left\|\tilde{d}_{i}\right\|_{\infty} \leq 1\right\}$ where $\tilde{n}_{i}=\sum_{j \in \mathcal{N}_{i}} n_{j}+r_{i} \Psi_{i}=\left[\begin{array}{llll}\bar{A}_{i j_{1}} \Xi_{j_{1}} & \ldots & \bar{A}_{i j_{z}} \Xi_{j_{z}} & D_{i} \Delta_{i}\end{array}\right], j_{1}, \ldots, j_{z} \in \mathcal{N}_{i}$.

Proposition 2. For given matrices $L_{i j}$ and parameters $\delta_{i j}, i, j \in \mathcal{M}$, if conditions (13) hold and

$$
\gamma_{i}=\sum_{k=0}^{\infty}\left\|\mathcal{H}_{i} \bar{A}_{i i}^{k} \Psi_{i}\right\|_{\infty}<1, \forall i \in \mathcal{M}
$$

then, there exists an RPI set $\mathbb{S}_{i} \subseteq \mathbb{E}_{i}$ for (14), such that $\mathbb{S}=\prod_{i \in \mathcal{M}} \mathbb{S}_{i}$ is an RPI set for system (111).

Proof. The proof is given in the Appendix 7.2

Remark 2. We note that if the subsystems are decoupled, then condition (16) implies that there exists an $m R P I \underline{\mathbb{S}}_{i} \subseteq \mathbb{E}_{i}$ for the local error dynamics (14). Moreover, when subsystems are coupled and $\mathbb{W}_{i}=\{0\}$, if $\beta_{i}<1$ then $\gamma_{i}<1$. Indeed, $\mathbb{W}_{i}=\{0\}$ implies that $\Delta_{i}=0$ and, as shown in the proof of Proposition 1, it holds $\sum_{k=0}^{\infty} \sum_{j \in \mathcal{N}_{i}}\left\|\mathcal{H}_{i} \bar{A}_{i i}^{k} \bar{A}_{i j} \Xi_{j}^{b}\right\|_{\infty} \leq \sum_{k=0}^{\infty} \sum_{j \in \mathcal{N}_{i}}\left\|\mathcal{H}_{i} \bar{A}_{i i}^{k} \bar{A}_{i j} \mathcal{H}_{j}^{b}\right\|_{\infty}$. Finally, the pieces of information needed for computing scalars $\gamma_{i}$ are the same needed for computing scalars $\beta_{i}$ (see Remark [1).

From results in Proposition 1 and 2. Problem 1 can be decomposed into the following independent design problems for $i \in \mathcal{M}$.

\section{Problem $\mathcal{P}_{i}$}

Check if there exist $L_{i i}$ and $\left\{L_{i j}\right\}_{j \in \mathcal{N}_{i}}$ such that $\bar{A}_{i i}$ is Schur, $\beta_{i}<1$ and $\gamma_{i}<1$.

Remark 3. As shown in [17], a necessary condition for the existence of RPI sets $\mathbb{S}_{i}$ for (14) is that

$$
\mathbb{E}_{i} \subseteq \tilde{\mathbb{V}}_{i}, \forall i \in \mathcal{M}
$$

where $\tilde{\mathbb{V}}_{i}$ depend upon sets $\mathbb{E}_{j}, j \in \mathcal{N}_{i}$, see (15). In our approach, sets $\mathbb{E}_{i}$ are assigned a priori on the basis, e.g. of application-dependent constraints. Therefore we implicitly assume conditions (17) are verified. However, if subsystems are added sequentially to an existing plant and LSEs are designed with the PnP procedure described in Section 4 . conditions (17) are automatically checked and, if violated, they prevent from plugging-in subsystem $\Sigma_{[i]}$. We also highlight that when sets $\mathbb{E}_{i}$ can be arbitrarily chosen, centralized methods for fulfilling conditions (17) exist [7].

\subsection{Optimization-based synthesis of LSEs}

The procedure for solving problems $\mathcal{P}_{i}, i \in \mathcal{M}$ is summarized in Algorithm 1 that can be executed in parallel by each subsystem using local hardware.

In step (11), if $\delta_{i j}=1$, the computation of matrices $L_{i j}, j \in \mathcal{N}_{i}$ is required. Since the choice of $L_{i j}$ affects the coupling term $\bar{A}_{i j}=A_{i j}+\delta_{i j} L_{i j} C_{j}$, and hence the possibility of verifying inequalities (13) and (16), we propose to reduce the magnitude of coupling by minimizing the 


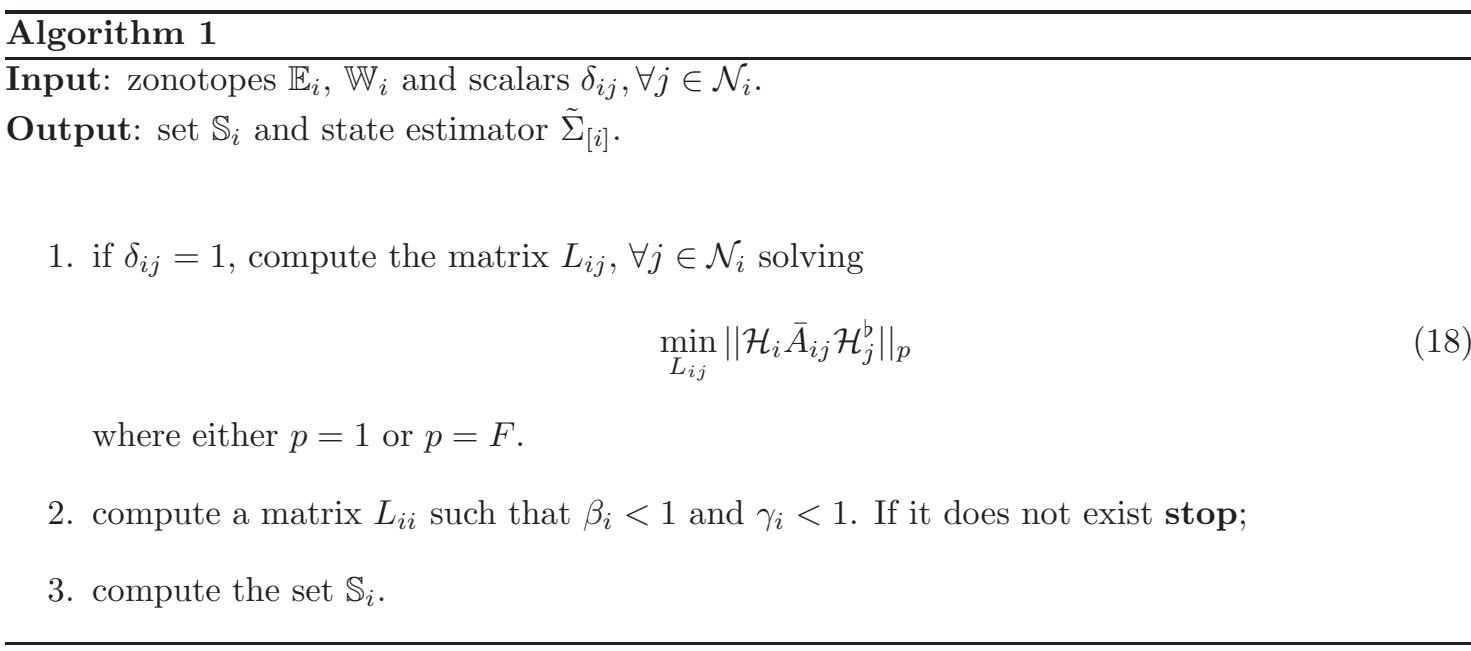

magnitude of $\bar{A}_{i j}$ in (18), where $\mathcal{H}_{i}$ and $\mathcal{H}_{j}^{b}$ allow us to take into account the size of sets $\mathbb{E}_{i}$ and $\mathbb{E}_{j}$, respectively. More precisely, it can be shown that the term $\left\|\mathcal{H}_{i} \bar{A}_{i j} \mathcal{H}_{j}^{b}\right\|_{p}$ is a measure of how much the coupling term $\bar{A}_{i j} e_{[j]}, j \in \mathcal{N}_{i}$ affects the fulfillment of the constraint $e_{[i]} \in \mathbb{E}_{i}$ (see Appendix (7.3). We highlight that the minimization of $\left\|\mathcal{H}_{i} \bar{A}_{i j} \mathcal{H}_{j}^{b}\right\|_{1}$ in (18) amounts to an LP problem and the minimization of $\left\|\mathcal{H}_{i} \bar{A}_{i j} \mathcal{H}_{j}^{b}\right\|_{F}$ can be recast into a Quadratic Programming (QP) problem. So far, the parameters $\delta_{i j}$ have been considered fixed. However, if in step (11) one obtains $L_{i j}=0$ for some $j \in \mathcal{N}_{i}$, it is impossible to reduce the magnitude of the coupling term $\bar{A}_{i j}$ and the knowledge of $y_{[j]}$ is useless for estimator $\tilde{\Sigma}_{[i]}$. This suggests to revise the choice of $\delta_{i j}$ and set $\delta_{i j}=0$.

In step (2), for the computation of matrix $L_{i i}$ we propose an automatic method in order to guarantee satisfaction of inequalities (13) and (16). This procedure parallels the method proposed in [9] for control design. Since in (13a) we require the Schurness of matrix $\bar{A}_{i i}$, we need to guarantee that $L_{i i}$ stabilizes the pair $\left(A_{i i}, C_{i}\right)$. In order to achieve this aim we design $L_{i i}$ as the dual LQ control gain associated to matrices $Q_{i} \geq 0$ and $R_{i}>0$, i.e.

$$
L_{i i}=\left(R_{i}+C_{i} \bar{P}_{i} C_{i}^{T}\right)^{-1} C_{i} \bar{P}_{i} A_{i i}^{T}
$$

where $\bar{P}_{i}$ is the solution to the algebraic Riccati equation

$$
A_{i i} \bar{P}_{i} A_{i i}^{T}+Q_{i}-A_{i i} \bar{P}_{i} C_{i}^{T}\left(R_{i}+C_{i} \bar{P}_{i} C_{i}^{T}\right)^{-1} C_{i} \bar{P}_{i} A_{i i}^{T}=\bar{P}_{i}
$$

We then solve the following nonlinear optimization problem

$$
\begin{aligned}
\min _{Q_{i}, R_{i}} & \beta_{i} \\
Q_{i} & \geq 0, R_{i}>0 \\
\beta_{i} & <1 \\
\gamma_{i} & <1
\end{aligned}
$$

where constraint (20d) is needed only if $\mathbb{W}_{i} \neq\{0\}$. In order to simplify the optimization problem (20) one can assume $Q_{i}=\operatorname{diag}\left(q_{i, 1}, \ldots, q_{i, n_{i}}\right), R_{i}=\operatorname{diag}\left(r_{i, 1}, \ldots, r_{i, m_{i}}\right)$ and replace the matrix inequalities in (20b) with the scalar inequalities $q_{i, k} \geq 0, k \in 1: n_{i}$ and $r_{i, k}>0, k \in 1: m_{i}$. The feasibility of problem (20) guarantees that the estimator $\tilde{\Sigma}_{[i]}$ can be successfully designed. Note 
that if all matrices $L_{i j}, j \in \mathcal{N}_{i}$ are such that $\bar{A}_{i j}=0$, the inequality (20c) is always fulfilled and, when $\mathbb{W}=\{0\}$, the optimization problem (20) is reduced to the solution of the algebraic Riccati equation (19).

In step (3) of Algorithm 1 we need to compute a nonempty RPI set $\mathbb{S}_{i} \subseteq \mathbb{E}_{i}$ that, in view of Propositions 1 and 2, exists if the optimization problem (20) is feasible. To this purpose, several algorithms can be used. For instance, [14 discusses the computation of $\epsilon$-outer approximation of the mRPI $\underline{\mathbb{S}}_{i}$. The MRPI set $\overline{\mathbb{S}}_{i}$ can be obtained using methods in [18. More recently, efficient procedures have been also proposed for computing polytopic [15] or zonotopic [19] RPI sets.

\section{Plug-and-play operations}

Consider a plant composed by subsystems $\Sigma_{[i]}, i \in \mathcal{M}$ equipped with local state estimators $\tilde{\Sigma}_{[i]}$, $i \in \mathcal{M}$ produced by Algorithm 1 In case subsystems are added or removed, we show how to preserve properties (8) and (9) by updating a limited number of existing LSEs. Note that plugging in and unplugging of subsystems are here considered as off-line operations, i.e. they do not lead to switching between different dynamics in real time.

\subsection{Plugging in operation}

We start considering the plugging in of subsystem $\Sigma_{[M+1]}$, characterized by parameters $A_{M+1, M+1}$, $C_{M+1}, \mathbb{E}_{M+1}, \mathbb{W}_{M+1}, \mathcal{N}_{M+1}$ and $\left\{A_{M+1, j}\right\}_{j \in \mathcal{N}_{M+1}}$. In particular, $\mathcal{N}_{M+1}$ identifies the subsystems that will influence $\Sigma_{[M+1]}$ through matrices $\left\{A_{M+1, j}\right\}_{j \in \mathcal{N}_{M+1}}$. Subsystems that will be influenced by $\Sigma_{[M+1]}$ are given by $\mathcal{S}_{M+1}$ where

$$
\mathcal{S}_{i}=\left\{j: i \in \mathcal{N}_{j}\right\}
$$

is the set of children of subsystem $\Sigma_{[i]}$. For designing the LSE $\tilde{\Sigma}_{[M+1]}$ we execute Algorithm $\square$ that needs information only from subsystems $\Sigma_{[j]}, j \in \mathcal{N}_{M+1}$. If Algorithm 1 stops before the last step, we declare that $\Sigma_{[M+1]}$ cannot be plugged in. Since sets $\mathcal{N}_{j}, j \in \mathcal{S}_{M+1}$ have now one more element, previously obtained matrices $L_{j j}, j \in \mathcal{S}_{M+1}$ might give $\beta_{i} \geq 1$ or $\gamma_{i} \geq 1$. Indeed, quantities $\beta_{i}$ and $\gamma_{i}$ in (13) and (16) can only increase. Furthermore, the size of the set $\mathbb{S}_{j}$ increases and therefore the condition $\mathbb{S}_{j} \subseteq \mathbb{E}_{j}$ could be violated. This means that for each $j \in \mathcal{S}_{M+1}$ the LSE $\tilde{\Sigma}_{[j]}$ must be redesigned by running Algorithm 1. Again, if Algorithm 1 stops before completion for some $j \in \mathcal{S}_{M+1}$, we declare that $\Sigma_{[M+1]}$ cannot be plugged in.

Note that LSE redesign does not propagate further in the network, i.e. even without changing state estimators $\tilde{\Sigma}_{[i]}, i \notin\{M+1\} \bigcup \mathcal{S}_{M+1}$, properties (8) and (9) are guaranteed for the new DSE.

\subsection{Unplugging operation}

We consider the unplugging of system $\Sigma_{[k]}, k \in \mathcal{M}$. Since for each $i \in \mathcal{S}_{k}$ the set $\mathcal{N}_{i}$ contains one element less, one has that $\beta_{i}$ in (13) and $\gamma_{i}$ in (16) cannot increase. Furthermore, the set $\mathbb{S}_{i}^{0}$, chosen before the removal of system $\Sigma_{[k]}$, still verifies $\mathbb{S}_{i}^{0} \supseteq \tilde{\mathbb{V}}_{i}$ and therefore previously obtained optimizers for problem (18) can still be used. This means that for each $i \in \mathcal{S}_{k}$ the LSE $\tilde{\Sigma}_{[i]}$ does not have to be redesigned. Moreover, since for each system $\Sigma_{[j]}, j \notin\{k\} \bigcup \mathcal{S}_{k}$, the set $\mathcal{N}_{j}$ does not change, the redesign of the LSE $\tilde{\Sigma}_{[j]}$ is not required.

In conclusion, the removal of system $\Sigma_{[k]}$ does not require the redesign of any LSE in order to 
guarantee (8) and (9). However systems $\Sigma_{[i]} i \in \mathcal{S}_{k}$ have one parent less and the redesign of LSEs $\tilde{\Sigma}_{[i]}$ through Algorithm 1 could improve the performance.

\section{Example}

We consider a system composed by 16 masses coupled as in Figure 2 where the four edges connected to a point correspond to springs and dampers arranged as in Figure 1 Each mass $f \in 1: 16$ is

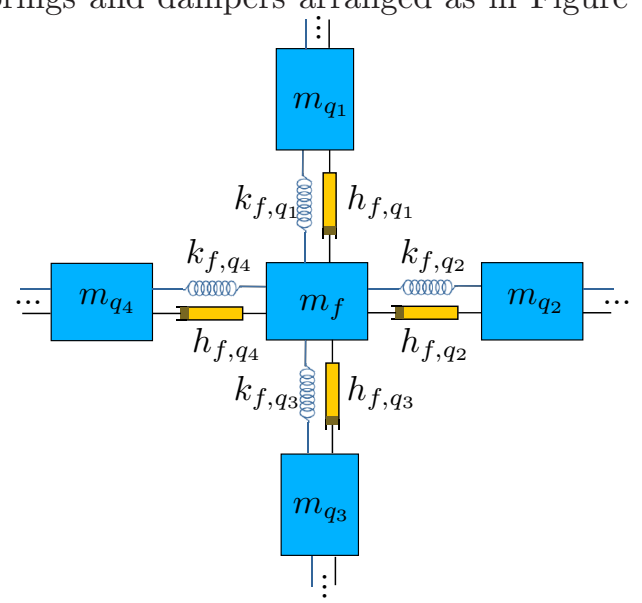

Figure 1: Array of masses: details of interconnections.

an LTI system with state variables $x_{[f]}=\left(x_{[f, 1]}, x_{[f, 2]}, x_{[f, 3]}, x_{[f, 4]}\right)$ and input $u_{[f]}=\left(u_{[f, 1]}, u_{[f, 2]}\right)$, where $x_{[f, 1]}$ and $x_{[f, 3]}$ are the displacements of mass $f$ with respect to a given equilibrium position in the plane (equilibria lie on a regular grid), $x_{[f, 2]}$ and $x_{[f, 4]}$ are the horizontal and vertical velocity of the mass $f$, respectively, and $100 u_{[f, 1]}$ (respectively $100 u_{[f, 2]}$ ) is the force applied to mass $f$ in the horizontal (respectively, vertical) direction. The values of $m_{f}$ have been extracted randomly in the interval $[5,10]$ while spring constants and damping coefficients are identical and equal to 0.5. Each mass is equipped with local state estimation error constraints $\left\|e_{[f, j]}\right\|_{\infty} \leq 1, j=1,3$ and $\left\|e_{[f, l]}\right\|_{\infty} \leq 1.5, l=2,4$.

A subsystem $\Sigma_{[i]}, i \in \mathcal{M}=1: 4$ is a group of four masses as in Figure 2] Therefore each subsystem has order 16 and two neighbors. For each subsystem $\Sigma_{[i]}$ we have 8 outputs that are the displacements of two masses and the velocities of the other two masses. We obtain models

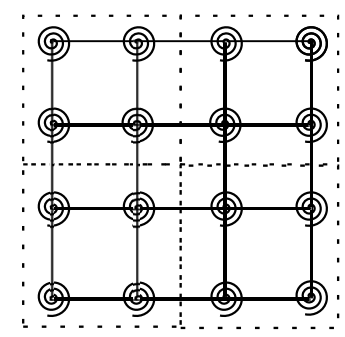

Figure 2: Position of the 16 masses on the plane. Dashed lines define subsystems $\Sigma_{[i]}, i \in \mathcal{M}=$ $1: 4$.

$\Sigma_{[i]}$ by discretizing continuous-time models with $0.2 \mathrm{sec}$ sampling time, using zero-order hold discretization for the local dynamics and treating $x_{[j]}, j \in \mathcal{N}_{i}$ as exogenous signals. We design 
an LSE $\tilde{\Sigma}_{[i]}, i \in \mathcal{M}$ using Algorithm 1 and assuming matrices $Q_{i}$ and $R_{i}$ in (20) are diagonal. In Figure 3 we show a simulation where the initial state of each mass is $x_{[f]}(0)=0, f \in 1: 16$ and the control inputs $u_{[f, l]}(k)=0.1 \sin (k), l \in 1: 2$, have been used. We initialize each LSE in order to have $e_{[i]} \in \mathbb{S}_{i}$. Estimation results produced by LSEs that have been designed with $\delta_{i j}=0, j \in \mathcal{N}_{i}$ are represented in Figures $3(\mathrm{a})$ and $3(\mathrm{c})$. Results obtained by setting $\delta_{i j}=1, j \in \mathcal{N}_{i}$ are shown in Figures $3(\mathrm{~b})$ and $3(\mathrm{~d})$. One can notice that in both cases, state estimation errors converge to zero and they are bounded at all times.

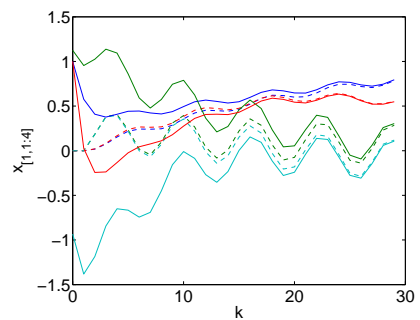

(a) State (dashed lines) and state estimation (continuous line) of the upper left mass in Figure 2 at time instants $k=0: 29$.

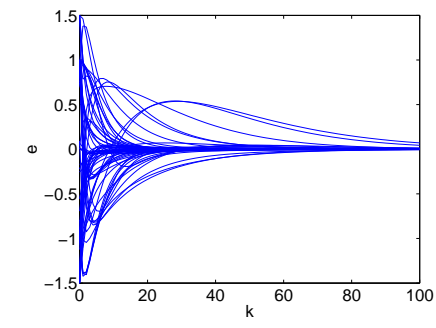

(c) Estimation errors for all states at times $k \in 0: 99$.

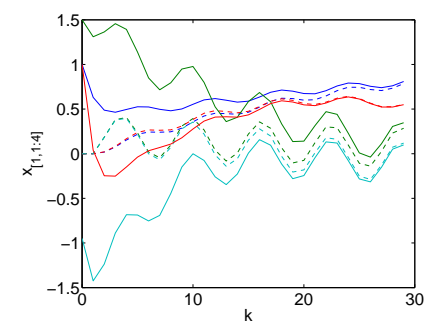

(b) State (dashed lines) and state estimation (continuous line) of the upper left mass in Figure 2 at time instants $k \in 0: 29$.

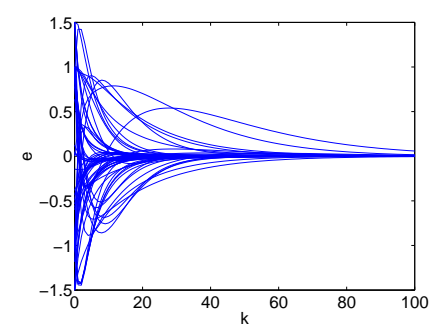

(d) Estimation errors for all states at times $k \in 0: 99$.

Figure 3: State estimation results for LSEs designed setting $\delta_{i j}=0, j \in \mathcal{N}_{i}$ (panels 3(a) and 3(c) and $\delta_{i j}=1, j \in \mathcal{N}_{i}$ (panels 3(b) and 3(d)]. In panels 3(a) and 3(b) the same color has been used for a state and its estimate: cyan and green lines denote velocities while blue and red lines denote positions.

In Figure 4 we show a simulation where each state of subsystem $\Sigma_{[i]}, i \in 1: 4$ is affected by a disturbance $w_{[i]}$ sampled from the uniform distribution in the set $\mathbb{W}_{i}=\left\{w_{[i]} \in \mathbb{R}:\left|w_{[i]}\right| \leq 0.015\right\}$. This has been obtained setting $D_{i}=\mathbf{1}_{16}$.

Figures 4(a) and 4(c) show results produced by LSEs designed with $\delta_{i j}=0, j \in \mathcal{N}_{i}$ while Figures 4(b) and 4(d) show the results obtained for $\delta_{i j}=1, j \in \mathcal{N}_{i}$. In both cases, errors fulfill the prescribed bounds but do not converge to zero because of the persistent disturbances $w_{[i]}$, $i \in 1: 4$.

\section{Conclusions}

We have proposed a novel DSE for large-scale linear perturbed systems, which guarantees that the estimation errors are bounded into prescribed sets and converge to zero in absence of disturbances. 


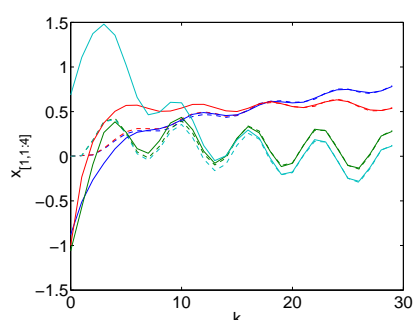

(a) State (dashed lines) and state estimation (continuous line) of the upper left mass in Figure 2 at time instants $k=0: 29$.

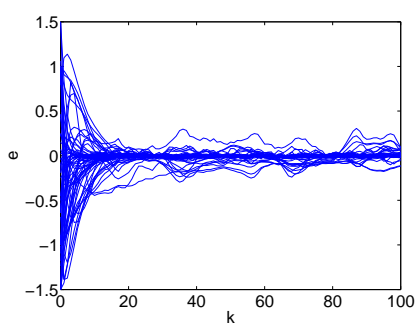

(c) Estimation errors for all states at times $k \in 0: 99$.

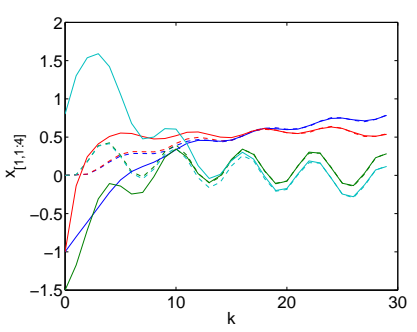

(b) State (dashed lines) and state estimation (continuous line) of the upper left mass in Figure 2 at time instants $k=0: 29$.

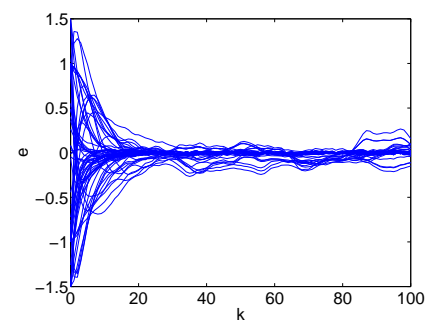

(d) Estimation errors for all states at times $k \in 0: 99$.

Figure 4: State estimation results for LSEs designed setting $\delta_{i j}=0, j \in \mathcal{N}_{i}$ (panels 4(a) and 4(c) and $\delta_{i j}=1, j \in \mathcal{N}_{i}$ (panels 4(b) and 4(d)p. In panels 4(a) and 4(b) the same color has been used for a state and its estimate: cyan and green lines denote velocities while blue and red lines denote positions.

The algorithm is based on the partition of the overall system into subsystems with non-overlapping states. In particular, the design of LSEs can be carried out in a decentralized fashion by solving a suitable optimization problem where just information by parent nodes is required. This allows one to efficiently update the overall DSE when subsystems are plugged in and out.

Future works include the design of output-feedback PnP schemes combining the state estimator proposed in this paper and the state-feedback $\mathrm{PnP}$ controller presented in 9 .

\section{$7 \quad$ Appendix}

\subsection{Proof of Proposition 1}

The proof uses arguments that are similar to the ones adopted for proving points (I) and (II) of Theorem 2 in 20 .

\subsubsection{Proof of (I)}

Define a matrix $\mathrm{IM}$ such that its $i j$-th entry $\mu_{i j}$ is

$$
\begin{array}{lll}
\mu_{i j}=-1 & \text { if } & i=j \\
\mu_{i j}=\sum_{k=0}^{\infty}\left\|\mathcal{H}_{i} \bar{A}_{i i}^{k} \bar{A}_{i j} \mathcal{H}_{j}^{b}\right\|_{\infty} & \text { if } & i \neq j .
\end{array}
$$


Note that all the off-diagonal entries of matrix $\mathbb{M}$ are non-negative, i.e., $\mathbb{M}$ is Metzler [21]. We recall the following results.

Lemma 1 (see 22]). Let matrix $\mathbb{I M} \in \mathbb{R}^{M \times M}$ be Metzler. Then $\mathbb{M}$ is Hurwitz if and only if there is a vector $\nu \in \mathbb{R}_{+}^{M}$ such that $\mathbb{M} \nu<\boldsymbol{O}_{M}$.

Lemma 2. Define the matrix $\Gamma=\mathbb{M}+\mathbb{I}_{M}$ where $\mathbb{M} \in \mathbb{R}^{M \times M}, \mathbb{I}_{M}$ is the $M \times M$ identity matrix and $\Gamma$ is non negative. Then the Metzler matrix $\mathbb{M}$ is Hurwitz if and only if $\Gamma$ is Schur.

The proof of Lemma 2 easily follows from Theorem 13 in 21 .

Inequalities (13) are equivalent to $\mathbb{M} \nu<\mathbf{0}_{M}$ where $\nu=\mathbf{1}_{M}$. Then, from Lemma $1 \mathrm{I}$ is Hurwitz. From Lemma 2. (13) implies that matrix $\Gamma=\mathbb{M}+\mathbb{I}_{M}$ is Schur.

For dynamics (12), we have

$$
e_{[i]}(t)=\bar{A}_{i i}^{t} e_{[i]}(0)+\sum_{k=0}^{t-1} \bar{A}_{i i}^{k} \sum_{j \in \mathcal{N}_{i}} \bar{A}_{i j} e_{[j]}(t-k-1)
$$

In view of (21) we can write

$$
\begin{aligned}
\left\|\mathcal{H}_{i} e_{[i]}(t)\right\|_{\infty} & \leq\left\|\mathcal{H}_{i} \bar{A}_{i i}^{t} \mathcal{H}_{i}^{b}\right\|_{\infty}\left\|\mathcal{H}_{i} e_{[i]}(0)\right\|_{\infty}+ \\
& +\sum_{j \in \mathcal{N}_{i}} \gamma_{i j} \max _{k \leq t}\left\|\mathcal{H}_{j} e_{[j]}(k)\right\|_{\infty}
\end{aligned}
$$

where $\gamma_{i j}$ are the entries of $\Gamma$. Denoting $\tilde{e}_{[i]}=\mathcal{H}_{i} e_{[i]}$, we can collectively define $\tilde{\mathbf{e}}=\tilde{\mathcal{H}} \mathbf{e}$, where $\tilde{\mathcal{H}}=\operatorname{diag}\left(\mathcal{H}_{1}, \ldots, \mathcal{H}_{M}\right)$. From the definition of sets $\mathbb{E}_{i}$, we have $\operatorname{rank}(\tilde{\mathcal{H}})=n$. We define the system

$$
\tilde{\mathbf{e}}^{+}=\tilde{\bar{A}} \tilde{\mathbf{e}}
$$

where $\tilde{\bar{A}}=\tilde{\mathcal{H}} \overline{\mathbf{A}} \tilde{\mathcal{H}}^{b}$. In order to analyze the stability of the origin of (22), we use the small gain theorem for networks in [16. In view of Corollary 16 in [16, the overall system (22) is asymptotically stable if the gain matrix $\Gamma$ is Schur and, as shown above, this property is implied by (13). Moreover, system (22) is an expansion of the original system (see Chapter 3.4 in 23]). In view of the inclusion principle [24, the asymptotic stability of (22) implies the asymptotic stability of the original system.

\subsubsection{Proof of (II)}

First note that, for $i \in \mathcal{M}$, since $\mathbb{E}_{i}$ is a zonotope, $\left\|h_{i, \tau}^{T} \Xi_{i}\right\|_{\infty}=1$ for all $\tau \in 1: \bar{\tau}_{i}$ and therefore $\left\|\mathcal{H}_{i} \Xi_{i}\right\|_{\infty}=1$. This implies that $\left\|h_{i, \tau}^{T} \bar{A}_{i i}^{k} \bar{A}_{i j} \Xi_{j}\right\|_{\infty} \leq\left\|h_{i, \tau}^{T} \bar{A}_{i i}^{k} \bar{A}_{i j} \mathcal{H}_{j}^{b}\right\|_{\infty}\left\|\mathcal{H}_{j} \Xi_{j}\right\|_{\infty}=$ $\left\|h_{i, \tau}^{T} \bar{A}_{i i}^{k} \bar{A}_{i j} \mathcal{H}_{j}^{b}\right\|_{\infty} \leq\left\|\mathcal{H}_{i} \bar{A}_{i i}^{k} \bar{A}_{i j} \mathcal{H}_{j}^{b}\right\|_{\infty}$.

Therefore, from (13b), for all $\tau \in 1: \bar{\tau}_{i}$ it holds

$$
\sum_{k=0}^{\infty} \sum_{j \in \mathcal{N}_{i}}\left\|h_{i, \tau}^{T} \bar{A}_{i i}^{k} \bar{A}_{i j} \Xi_{j}\right\|_{\infty} \leq \sum_{k=0}^{\infty} \sum_{j \in \mathcal{N}_{i}}\left\|\mathcal{H}_{i} \bar{A}_{i i}^{k} \bar{A}_{i j} \mathcal{H}_{j}^{b}\right\|_{\infty}<1
$$

The next aim is to prove that there exists an RPI $\mathbb{S}_{i} \subseteq \mathbb{E}_{i}$ for the dynamics (12), in particular we define $\mathbb{S}_{i}$ as an outer approximation of the $\operatorname{mRPI} \underline{\mathbb{S}}_{i}$ and we prove that the outer approximation 
always exists.

The mRPI for (12) is given by [14]

$$
\underline{\mathbb{S}}_{i}=\bigoplus_{k=0}^{\infty} \bar{A}_{i i}^{k} \bigoplus_{j \in \mathcal{N}_{i}} \bar{A}_{i j} \mathbb{E}_{j}
$$

From [14, for given $\epsilon_{i}>0$ there exist $\alpha_{i} \in \mathbb{R}$ and $s_{i} \in \mathbb{N}_{+}$such that the set

$$
\mathbb{S}_{i}\left(\epsilon_{i}\right)=\left(1-\epsilon_{i}\right)^{-1} \bigoplus_{k=0}^{s_{i}-1} \bar{A}_{i i}^{k} \bigoplus_{j \in \mathcal{N}_{i}} \bar{A}_{i j} \mathbb{E}_{j}
$$

is an $\epsilon_{i}$-outer approximation of the mRPI $\underline{\mathbb{S}}_{i}$.

Using arguments from Section 3 of [17, we can then guarantee that $\mathbb{S}_{i}\left(\epsilon_{i}\right) \subseteq \mathbb{E}_{i}$. In fact for all $\tau \in 1: \bar{\tau}_{i}$

$$
\sup _{s_{[i]} \in \mathbb{S}_{i}\left(\epsilon_{i}\right)} h_{i, \tau}^{T} s_{[i]} \leq 1 .
$$

Using (24), the inequalities (26) are verified if

$$
\sup _{\substack{\left\{e_{[j]}(k) \in \mathbb{E}_{j}\right\}_{j}^{k=0, \ldots, \infty} \\ \sigma_{i} \in B_{\epsilon_{i}}(0)}} z_{i, \tau}^{x}\left(\left\{e_{[j]}(k)\right\}_{j \in \mathcal{N}_{i}}^{k=0, \ldots, \infty}\right)+\left\|h_{i, \tau}^{T} \sigma_{i}\right\|_{\infty} \leq 1
$$

where $z_{i, \tau}^{x}(\cdot)=h_{i, \tau}^{T} \sum_{k=0}^{\infty} \bar{A}_{i i}^{k} \sum_{j \in \mathcal{N}_{i}} \bar{A}_{i j} e_{[j]}(k)$.

Since $\left\|h_{i, r}^{T} \sigma_{i}\right\|_{\infty} \leq\left\|h_{i, r}^{T}\right\|_{\infty} \epsilon_{i}$, conditions (27) are satisfied if

$$
\sup _{\left\{e_{[j]}(k) \in \mathbb{S}_{j}\right\}_{j \in \mathcal{N}_{i}}^{k=0, \ldots, \infty}} z_{i, \tau}^{x}\left(\left\{e_{[j]}(k)\right\}_{j \in \mathcal{N}_{i}}^{k=0, \ldots, \infty}\right) \leq 1-\left\|h_{i, \tau}^{T}\right\|_{\infty} \epsilon_{i} .
$$

Using (10), we can rewrite (28) as

$$
\sup _{\left\{\left\|d_{j}(k)\right\|_{\infty} \leq 1\right\}_{j \in \mathcal{N}_{i}}^{k=\ldots, \ldots}} z_{i, \tau}^{d}\left(\left\{d_{j}(k)\right\}_{j \in \mathcal{N}_{i}}^{k=0, \ldots, \infty}\right) \leq 1-\left\|h_{i, r}^{T}\right\|_{\infty} \epsilon_{i}
$$

where $z_{i, \tau}^{d}(\cdot)=h_{i, \tau}^{T}\left(\sum_{k=0}^{\infty} \bar{A}_{i i}^{k} \sum_{j \in \mathcal{N}_{i}} \bar{A}_{i j} \Xi_{j} d_{j}(k)\right)$.

The inequalities (29) are satisfied if

$$
\sum_{k=0}^{\infty} \sum_{j \in \mathcal{N}_{i}}\left\|h_{i, \tau}^{T} \bar{A}_{i i}^{k} \bar{A}_{i j} \Xi_{j}\right\|_{\infty} \leq 1-\left\|h_{i, \tau}^{T}\right\|_{\infty} \epsilon_{i}
$$

for all $\tau \in 1: \bar{\tau}_{i}$.

In view of (23), there exists a sufficiently small $\epsilon_{i}>0$ satisfying (30). Hence we proved that $\forall i \in \mathcal{M}$ there exists an RPI $\mathbb{S}_{i} \subseteq \mathbb{E}_{i}$ for dynamics (12). Moreover if we define $\mathbb{S}=\prod_{i \in \mathcal{M}} \mathbb{S}_{i}$, the set $\mathbb{S}$ is an invariant set for system (11) equipped with constraints $\mathbb{E}$ and $\mathbb{W}=\{0\}$.

\subsection{Proof of Proposition 2}

In the following we use similar arguments of Proof of Proposition 1 (see Section 7.1.2) to prove that there exists an RPI $\mathbb{S}_{i} \subseteq \mathbb{E}_{i}$ for the dynamics (14), in particular we define $\mathbb{S}_{i}$ as an outer approximation of the $\operatorname{mRPI} \underline{\mathbb{S}}_{i}$ and we prove that the outer approximation always exists.

The mRPI for (14) is given by [14]

$$
\underline{\mathbb{S}}_{i}=\bigoplus_{k=0}^{\infty} \bar{A}_{i i}^{k}\left(\bigoplus_{j \in \mathcal{N}_{i}} \bar{A}_{i j} \mathbb{E}_{j} \oplus D_{i} \mathbb{W}_{i}\right)=\bigoplus_{k=0}^{\infty} \bar{A}_{i i}^{k} \tilde{\mathbb{V}}_{i}
$$


From [14], for given $\epsilon_{i}>0$ there exist $\alpha_{i} \in \mathbb{R}$ and $s_{i} \in \mathbb{N}_{+}$such that the set

$$
\mathbb{S}_{i}\left(\epsilon_{i}\right)=\left(1-\epsilon_{i}\right)^{-1} \bigoplus_{k=0}^{s_{i}-1} \bar{A}_{i i}^{k} \tilde{\mathbb{V}}_{i}
$$

is an $\epsilon_{i}$-outer approximation of the mRPI $\underline{\mathbb{S}}_{i}$.

Using arguments from Section 3 of [17, we can then guarantee that $\mathbb{S}_{i}\left(\epsilon_{i}\right) \subseteq \mathbb{E}_{i}$. In fact for all $\tau \in 1: \bar{\tau}_{i}$

$$
\sup _{s_{[i]} \in \mathbb{S}_{i}\left(\epsilon_{i}\right)} h_{i, \tau}^{T} s_{[i]} \leq 1 .
$$

Using (31), the inequalities (33) are verified if

$$
\sup _{\substack{\sigma_{i} \in B_{\epsilon_{i}}(0) \\ \tilde{v}_{[i]} \in \mathbb{V}_{i}}} z_{i, \tau}^{x}\left(\left\{\tilde{v}_{[i]}(k)\right\}^{k=0, \ldots, \infty}\right)+\left\|h_{i, \tau}^{T} \sigma_{i}\right\|_{\infty} \leq 1
$$

where $z_{i, \tau}^{x}(\cdot)=h_{i, \tau}^{T} \sum_{k=0}^{\infty} \bar{A}_{i i}^{k} \tilde{v}_{[i]}$.

Since $\left\|h_{i, r}^{T} \sigma_{i}\right\|_{\infty} \leq\left\|h_{i, r}^{T}\right\|_{\infty} \epsilon_{i}$, conditions (27) are satisfied if

$$
\sup _{\tilde{v}_{[i]} \in \tilde{\mathbb{V}}_{i}} z_{i, \tau}^{x}\left(\left\{\tilde{v}_{[i]}(k)\right\}^{k=0, \ldots, \infty}\right) \leq 1-\left\|h_{i, \tau}^{T}\right\|_{\infty} \epsilon_{i}
$$

Using (10) and (44), we can rewrite (35) as

$$
\sup _{\left\{\left\|\tilde{d}_{i}(k)\right\|_{\infty} \leq 1\right\}^{k=0, \ldots, \infty}} z_{i, \tau}^{d}\left(\left\{\tilde{d}_{i}(k)\right\}^{k=0, \ldots, \infty}\right) \leq 1-\left\|h_{i, r}^{T}\right\|_{\infty} \epsilon_{i}
$$

where $z_{i, \tau}^{d}(\cdot)=h_{i, \tau}^{T}\left(\sum_{k=0}^{\infty} \bar{A}_{i i}^{k} \Psi_{i} \tilde{d}_{i}(k)\right)$.

The inequalities (36) are satisfied if

$$
\sum_{k=0}^{\infty}\left\|h_{i, \tau}^{T} \bar{A}_{i i}^{k} \Psi_{i}\right\|_{\infty} \leq 1-\left\|h_{i, \tau}^{T}\right\|_{\infty} \epsilon_{i}
$$

for all $\tau \in 1: \bar{\tau}_{i}$.

We proved that $\forall i \in \mathcal{M}$ there exists an RPI $\mathbb{S}_{i} \subseteq \mathbb{E}_{i}$ for dynamics (14). Moreover if we define $\mathbb{S}=\prod_{i \in \mathcal{M}} \mathbb{S}_{i}$, the set $\mathbb{S}$ is an RPI invariant set for system (11) equipped with constraints $\mathbb{E}$ and $\mathbb{W} \neq\{0\}$.

\subsection{Notes on the optimization problem (18)}

In order to fulfill condition (13b), we need to guarantee at least that

$$
\bar{A}_{i j} \mathbb{E}_{j} \subseteq \mathbb{E}_{i}
$$

hence

$$
\mathcal{H}_{i} \bar{A}_{i j} e_{[j]} \leq 1, \forall e_{[j]} \in \mathbb{E}_{j} .
$$

In order to minimize the effect of coupling terms $\bar{A}_{i j}$, from (10) we can solve the following optimization problem.

$$
\eta_{i j}=\min _{L_{i j}} \max _{\substack{[j] \\ \text { Ij] } \\\left\|d_{j}\right\|_{\infty} \leq 1}}\left\|\mathcal{H}_{i} \bar{A}_{i j} e_{[j]}\right\|_{p}
$$


where $p=1$ or $p=F$. Using arguments similar to the ones adopted in the proof of Proposition 1. from (38) we obtain

$$
\begin{aligned}
\eta_{i j} & \leq \min _{L_{i j}} \max _{\left\|d_{j}\right\|_{\infty} \leq 1}\left\|\mathcal{H}_{i} \bar{A}_{i j} \Xi_{j} d_{j}\right\|_{p} \\
& \leq \min _{L_{i j}} \max _{d_{j} \|_{\infty} \leq 1}\left\|\mathcal{H}_{i} \bar{A}_{i j} \mathcal{H}_{j}^{b}\right\|_{p}\left\|\mathcal{H}_{j} \Xi_{j} d_{j}\right\|_{p} \\
& \leq \min _{L_{i j}} \max _{\left\|d_{j}\right\|_{\infty} \leq 1}\left\|\mathcal{H}_{i} \bar{A}_{i j} \mathcal{H}_{j}^{b}\right\|_{p}\left\|\mathcal{H}_{j} \Xi_{j}\right\|_{p}\left\|d_{j}\right\|_{p}
\end{aligned}
$$

Irrespectively of $p$, there exist constants $c_{1, p}>0$ and $c_{2, p}>0$ such that

$$
\begin{gathered}
\left\|\mathcal{H}_{j} \Xi_{j}\right\|_{p} \leq c_{1, p}\left\|\mathcal{H}_{j} \Xi_{j}\right\|_{\infty}=c_{1, p} \\
\max _{\left\|d_{j}\right\|_{\infty} \leq 1}\left\|d_{j}\right\|_{p} \leq \max _{\left\|d_{j}\right\|_{\infty} \leq 1} c_{2, p}\left\|d_{j}\right\|_{\infty}=c_{2, p}
\end{gathered}
$$

Therefore, we can conclude that

$$
\eta_{i j} \leq c_{1, p} c_{2, p} \min _{L_{i j}}\left\|\mathcal{H}_{i} \bar{A}_{i j} \mathcal{H}_{j}^{b}\right\|_{p}
$$

and this motivates the optimization problem (18).

\section{References}

[1] A. G. O. Mutambara, Decentralized estimation and control for multisensor systems. Boca Raton, FL: CRC Press, 1998.

[2] R. Vadigepalli and F. J. Doyle III, "A distributed state estimation and control algorithm for plantwide processes," IEEE Transactions on Control Systems Technology, vol. 11, no. 1, pp. 119-127, 2003.

[3] U. A. Khan and J. M. F. Moura, "Distributing the Kalman Filter for Large-Scale Systems," IEEE Transactions on Signal Processing, vol. 56, no. 10, pp. 4919-4935, 2008.

[4] S. S. Stankovic, M. S. Stankovic, and D. M. Stipanovic, "Consensus based overlapping decentralized estimator," IEEE Transactions on Automatic Control, vol. 54, no. 2, pp. 410-415, 2009 .

[5] — , "Consensus based overlapping decentralized estimation with missing observations and communication faults," Automatica, vol. 45, pp. 1397-1406, 2009.

[6] M. Farina, G. Ferrari-Trecate, and R. Scattolini, "Moving-horizon partition-based state estimation of large-scale systems," Automatica, vol. 46, no. 5, pp. 910-918, 2010.

[7] M. Farina and R. Scattolini, "An output feedback distributed predictive control algorithm," in Proceedings of the 50th IEEE Conference on Decision and Control, and the European Control Conference, Orlando, FL, USA, December 12-15, 2011, pp. 8139-8144.

[8] S. Riverso, D. Rubini, and G. Ferrari-Trecate, "Distributed bounded-error state estimation for partitioned systems based on practical robust positive invariance," in Proceedings of the 12th European Control Conference, Zurich, Switzerland, July 17-19, 2013, pp. 2633-2638. 
[9] S. Riverso, M. Farina, and G. Ferrari-Trecate, "Plug-and-Play Decentralized Model Predictive Control for Linear Systems," IEEE Transactions on Automatic Control, p. In press, 2013. [Online]. Available: 10.1109/TAC.2013.2254641

[10] — , "Plug-and-Play Model Predictive Control based on robust control invariant sets," Dipartimento di Ingegneria Industriale e dell'Informazione, Universita' degli Studi di Pavia, Tech. Rep., 2012. [Online]. Available: arXiv:1210.6927

[11] T. Samad and T. Parisini, "Systems of Systems," in The Impact of Control Technology, T. Samad and A. M. Annaswamy, Eds. IEEE Control Systems Society, 2011, pp. 175-183. [Online]. Available: ieeecss.org/general/impact-control-technology

[12] P. J. Antsaklis, B. Goodwine, V. Gupta, M. J. McCourt, Y. Wang, P. Wu, M. Xia, H. Yu, and F. Zhu, "Control of cyberphysical systems using passivity and dissipativity based methods," European Journal of Control, vol. 19, no. 5, pp. 379-388, 2013.

[13] J. B. Rawlings and D. Q. Mayne, Model Predictive Control: Theory and Design. Madison, WI, USA: Nob Hill Pub., 2009.

[14] S. V. Raković, E. C. Kerrigan, K. I. Kouramas, and D. Q. Mayne, "Invariant approximations of the minimal robust positively invariant set," IEEE Transactions on Automatic Control, vol. 50, no. 3, pp. 406-410, 2005.

[15] S. V. Raković and M. Baric, "Parameterized Robust Control Invariant Sets for Linear Systems: Theoretical Advances and Computational Remarks," IEEE Transactions on Automatic Control, vol. 55, no. 7, pp. 1599-1614, 2010.

[16] S. Dashkovskiy, B. S. Rüffer, and F. R. Wirth, "An ISS small gain theorem for general networks," Mathematics of Control, Signals, and Systems, vol. 19, no. 2, pp. 93-122, 2007.

[17] I. Kolmanovsky and E. G. Gilbert, "Theory and computation of disturbance invariant sets for discrete-time linear systems," Mathematical Problems in Engineering, vol. 4, no. 4, pp. 317-363, 1998.

[18] E. G. Gilbert and K. T. Tan, "Linear Systems with State and Control Constraints: The Theory and Application of Maximal Output Admissible Sets," IEEE Transactions on Automatic Control, vol. 36, no. 9, pp. 1008-1020, 1991.

[19] S. V. Raković, "Robust Control of Constrained Discrete Time Systems: Characterization and Implementation," Ph.D. dissertation, Imperial College London, University of London, 2005.

[20] S. Riverso, M. Farina, and G. Ferrari-Trecate, "Plug-and-Play Decentralized Model Predictive Control," Dipartimento di Ingegneria Industriale e dell'Informazione, Universita' degli Studi di Pavia, Tech. Rep., 2012. [Online]. Available: arXiv:1302.0226

[21] L. Farina and S. Rinaldi, Positive Linear Systems. New York. NY, USA: John Wiley \& Sons, 2000.

[22] O. Mason and R. Shorten, "On Linear Copositive Lyapunov Functions and the Stability of Switched Positive Linear Systems," IEEE Transactions on Automatic Control, vol. 52, no. 7, pp. 1346-1349, 2007. 
[23] J. Lunze, Feedback control of large scale systems. Upper Saddle River, NJ, USA: Prentice Hall, Systems and Control Engineering, 1992.

[24] S. S. Stankovic, "Inclusion Principle for Discrete-Time Time-Varying Systems," Dynamics of Continuous, Discrete and Impulsive Systems, vol. 11, no. Series A: Mathematical Analysis, pp. 321-338, 2004. 